Neutron Monitors and Cosmogenic Isotopes as Cosmic Ray

Energy-Integration Detectors : Effective Yield Functions, Effective Energy, and Its Dependence on the Local Interstellar Spectrum

\title{
Asvestari, Eleanna
}

2017-10-13

Asvestari , E , Gil , A , Kovaltsov , G A \& Usoskin , I G 2017 , ' Neutron Monitors and Cosmogenic Isotopes as Cosmic Ray Energy-Integration Detectors : Effective Yield Functions, Effective Energy, and Its Dependence on the Local Interstellar Spectrum ' , Journal of geophysical research. Space physics , vol. 122 , no. 10 , pp. 9790 - 9802 . https://doi.org/10.1002/2017JA

cc_by_nc_sa

publishedVersion

Downloaded from Helda, University of Helsinki institutional repository.

This is an electronic reprint of the original article.

This reprint may differ from the original in pagination and typographic detail.

Please cite the original version. 


\section{Journal of Geophysical Research: Space Physics}

\section{RESEARCH ARTICLE \\ 10.1002/2017JA024469 \\ Key Points: \\ - Yield functions of realistic production/ deposition of cosmogenic isotopes \\ Neutron Monitors and Cosmogenic Isotopes as Cosmic Ray Energy-Integration Detectors: Effective Yield Functions, Effective Energy, and Its Dependence on the Local Interstellar Spectrum}

${ }^{14} \mathrm{C}$ and ${ }^{10} \mathrm{Be}$ are presented

- Effective energy of neutron monitors,

${ }^{14} \mathrm{C}$ and ${ }^{10} \mathrm{Be}$ is calculated

- Conversion of the modulation

potential values between different

LIS models is performed

Supporting Information:

- Table S1

Correspondence to:

I. G. Usoskin,

ilya.usoskin@oulu.fi

Citation:

Asvestari, E., Gil, A., Kovaltsov, G. A., \& Usoskin, I. G. (2017). Neutron monitors and cosmogenic isotopes as cosmic ray energy-integration detectors: Effective yield functions, effective energy, and its dependence on the local interstellar spectrum. Journal of Geophysical Research: Space Physics, 122, 9790-9802. https://doi.org/10.1002/2017JA024469

Received 13 JUN 2017 Accepted 11 SEP 2017

Accepted article online 18 SEP 2017 Published online 13 OCT 2017

(02017. American Geophysical Union All Rights Reserved.

\author{
Eleanna Asvestari' ${ }^{1,2}$ (D) , Agnieszka Gil ${ }^{3}$ iD , Gennady A. Kovaltsov ${ }^{4}$, and Ilya G. Usoskin ${ }^{1,5}$ (D) \\ ${ }^{1}$ Space Climate Research Unit, University of Oulu, Oulu, Finland, ${ }^{2}$ Department of Physics, University of Helsinki, Helsinki, \\ Finland, ${ }^{3}$ Institute of Mathematics and Physics, Siedlce University, Siedlce, Poland, ${ }^{4}$ loffe Physical-Technical Institute, \\ St. Petersburg, Russia, ${ }^{5}$ Sodankylä Geophysical Observatory, University of Oulu, Oulu, Finland
}

\section{Introduction}

For many purposes, both academic and practical, it is important to know the variability of the near-Earth radiation environment, which is mostly defined, at least in the higher-energy range above several hundred $\mathrm{MeV}$, by galactic cosmic rays (GCR). While the GCR flux can be assumed roughly constant (over reasonable timescales of up to tens of thousand years) beyond the solar system, the energy spectrum of GCR varies near the Earth because of its modulation by solar magnetic activity within solar cycle (Jokipii and Kóta, 1995; Jokipii and Levy, 1977; Potgieter, 2013). The depth of the cyclic variability depends on the particles' energy from less than a percent at tens of $\mathrm{GeV}$ to 2 orders of magnitude in the lower energy range $(\approx 100 \mathrm{MeV})$. Usually, no modulation is assumed for GCR with energy exceeding $100 \mathrm{GeV}$.

The GCR energy spectrum can be measured near Earth by balloon-borne or spaceborne instruments. Measurements are regular since 2006 when precise spectrometers PAMELA (Adriani et al., 2013) and later AMS-02 (Aguilar et al., 2015) were launched to a low orbit. Before that, direct data of GCR spectrum were only available from fragmentary balloon-borne campaigns (e.g., Abe et al., 2016) and a short AMS-01 flight in 1998 (Alcaraz et al., 2000). The GCR variability near Earth is permanently monitored by the global network of ground-based neutron monitors (Dorman, 2004), which is an energy-integrating device and cannot resolve the energy spectrum. Neutron monitors (NMs) are in operation since the early 1950s, providing a systematic data set of cosmic ray variability. Before that, there are data from ground-based and balloon-borne ionization chambers since the mid-1930s (McCracken \& Beer, 2007) but they still may contain a long-term drift. Before the twentieth century, GCR variability can be reconstructed using measurements of cosmogenic radioisotopes in natural archives (Beer et al., 2012; Usoskin, 2017). The method of cosmogenic isotope also leads to an effective energy integration over the GCR spectrum and cannot resolve the detail of the spectrum. In this sense, 
cosmogenic isotopes $\left({ }^{14} \mathrm{C}\right.$ in tree rings and ${ }^{10} \mathrm{Be}$ in polar ices) also can be considered as energy-integrating devices, similar to a NM.

It has been found that the so-called force-field model of GCR heliospheric modulation (Gleeson \& Axford, 1968) provides a very handy parametrization of the GCR energy spectrum with a single time-variable parameter, the heliospheric modulation potential (Caballero-Lopez \& Moraal, 2004; Vainio et al., 2009). Thus, even a simple energy-integrating ground-based detector can monitor the GCR spectrum variability in the framework of the force-field approximation.

However, the exact value of the modulation potential reconstructed from the NM or cosmogenic isotope data depends on the assumption of the local interstellar spectrum (LIS) of GCR, viz., energy spectrum beyond the solar system. While the shape of LIS is directly measured in the low-energy part (below $100 \mathrm{MeV}$ ), thanks to the Voyager missions outside the heliospheric termination shock (Stone et al., 2013), and the higher-energy part (above $100 \mathrm{GeV}$ ) is not modulated in the heliosphere, the spectrum is not well constrained in the middle-energy part where solar modulation is crucial. On the other hand, the exact value of the modulation potential depends strongly on the LIS model used (Herbst et al., 2010; Maurin et al., 2015; Usoskin et al., 2005). Accordingly, there is a handful of different LIS models, which calls for a need to "intercalibrate" the modulation potential values, reconstructed from ground-based detectors. Because of this, the values of the modulation potential obtained by different methods and groups cannot be compared directly without a proper conversion, which is, however, not well defined yet.

In this work we revisit the method of assessment of GCR variability over different timescales using energyintegrating detectors such as a NM and cosmogenic isotopes ${ }^{10} \mathrm{Be}$ and ${ }^{14} \mathrm{C}$ stored in natural archives. Section 2 presents formalism to describe the energy spectrum of GCR in the vicinity of Earth, including effective yield functions for globally mixed ${ }^{14} \mathrm{C}$ and realistic deposition of ${ }^{10} \mathrm{Be}$. Details of the GCR measurements by an energy-integrating device are provided in section 3. A new concept of the effective energy of a detector is introduced in section 4. A problem of different LIS models is addressed in section 5. Conclusions are summarized in section 6 .

\section{GCR Near Earth}

\subsection{Force-Field Approximation}

The differential energy spectrum of GCR at the Earth's orbit is often described by the force-field approximation (e.g., Caballero-Lopez \& Moraal, 2004; Gleeson \& Axford, 1968; Usoskin et al., 2005) so that the energy spectrum of the $i$ th type of GCR particles, with mass $A_{i}$ and charge $Z_{i}$ numbers, can be described as follows:

$$
J_{i}(T)=J_{\mathrm{LIS}_{i}}\left(T+\Phi_{i}\right) \frac{E^{2}-T_{r}^{2}}{\left(E+\Phi_{i}\right)^{2}-T_{r}^{2}},
$$

where $J_{\mathrm{LS}_{i}}$ is the unmodulated local interstellar spectrum (LIS), $E=T+T_{r}$ is the total particle's energy per nucleon, which is the sum of kinetic energy per nucleon, $T$, and proton's rest mass energy, $T_{r}=0.938 \mathrm{GeV}, \Phi_{i}$ is defined by the modulation potential $\phi$, as $\Phi_{i}=\phi \cdot\left(e Z_{i} / A_{i}\right)$. This approach is widely used since the $1960 \mathrm{~s}$ in many applications such as terrestrial effects of cosmic rays, cosmogenic isotopes, lunar, and meteoritic studies (e.g., Asvestari et al., 2017; Inceoglu et al., 2015; Michel et al., 1996; Pallé et al., 2004; Webber \& Higbie, 2003). We note that this formula does not imply any physical insight into the heliospheric modulation but just provides a very useful parametrization of the cosmic ray (CR) spectrum. Accordingly, the absolute value of the modulation potential $\phi$ should be considered only as a parameter without a clear physical meaning. Moreover, a proper conversion is needed between $\phi$ values obtained by different methods and groups.

\subsection{Local Interstellar Spectra}

Unfortunately, our knowledge of the LIS is incomplete, leading to a diversity of the LIS estimates. The shape of LIS is relatively well know in the high-energy range above $100 \mathrm{GeV} /$ nucleon, where there is hardly any heliospheric modulation, and the spectrum can be directly assessed from near-Earth and ground-based measurements. The low-energy part (below a few hundred $\mathrm{MeV} /$ nucleon) of LIS is measured by Voyager spacecraft beyond the heliospheric termination shock (Stone et al., 2013) and is also relatively well known. The middle-energy part is, however, not well constrained since it is significantly affected by the heliospheric modulation process. It is usually estimated through "demodulation" of the near-Earth measurements back to the interstellar medium and applying a model of galactic transport to a theoretically expected GCR accelerated spectrum (e.g., Moskalenko et al., 2002). 


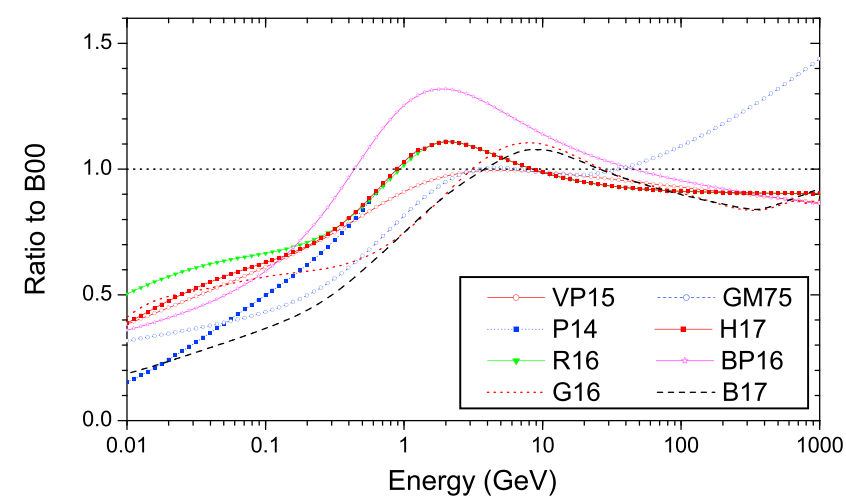

Figure 1. The ratio of different proton LIS approximations (as denoted in the legend) used here to that of B00. Spectra are denoted as discussed in section 2.2.
In earlier works, before direct data from Voyager and AMS and PAMELA, the basic LIS was often taken in the form of GM75 and B00 (see below). Several attempts have been made recently to revisit the shape of LIS, based on the most recent data sets. However, there are still essential discrepancies in the LIS shape in the middle-energy range as illustrated in Figure 1. Here we consider seven recent and two earlier LIS reconstructions for protons as described below (all spectra are given in units of (particles/( $\mathrm{m}^{2} \mathrm{sr} \mathrm{s} \mathrm{GeV} /$ nucleon)) and energy in $\mathrm{GeV})$ :

2.2.1. LIS by Potgieter et al. (2014): P14

$$
J_{\mathrm{LIS}_{\mathrm{P} 14}}(T)= \begin{cases}707 \times \exp \left(4.64-0.08 \cdot(\ln (T))^{2}-2.91 \cdot T^{0.5}\right) & \mathrm{T}<1.4 \mathrm{GeV} \\ 685 \times \exp \left(3.22-2.78 \cdot \ln (T)-1.5 \cdot T^{-1}\right) & \mathrm{T} \geq 1.4 \mathrm{GeV}\end{cases}
$$

\subsubsection{LIS by Vos and Potgieter (2015): VP15}

$$
J_{\mathrm{LIS}}(T)=\frac{2700 \cdot T^{1.12}}{\beta^{2}}\left(\frac{T+0.67}{1.67}\right)^{-3.93}
$$

where $\beta$ is the ratio of the proton's velocity $v$ per the speed of light.

2.2.3. LIS by Bisschoff and Potgieter (2016): BP16

$$
J_{\mathrm{LIS}_{\mathrm{BP} 16}}(T)=\frac{3719 \cdot T^{1.03}}{\beta^{2}} \cdot\left(\frac{T^{1.21}+0.77^{1.21}}{1+0.77^{1.21}}\right)^{-3.18} .
$$

2.2.4. LIS by Raath et al. (2016): R16

$$
J_{\mathrm{LIS}_{\mathrm{R} 16}}(T)= \begin{cases}697.8 \times \exp \left(4.64-0.023 \cdot(\ln (T))^{2}-2.91 \cdot T^{0.5}\right) & \mathrm{T}<1.4 \mathrm{GeV} \\ 684.7 \times \exp \left(3.22-2.78 \cdot \ln (T)-1.5 \cdot T^{-1}\right) & \mathrm{T} \geq 1.4 \mathrm{GeV}\end{cases}
$$

\subsubsection{LIS by Herbst et al. (2017): $\mathrm{H} 17$}

The model P14 was modified by $\mathrm{H} 17$ in the low-energy range, while they are identical for energies above $1.4 \mathrm{GeV}$.

$$
J_{\mathrm{LIS}_{\mathrm{H} 17}}(T)= \begin{cases}707 \times \exp \left(4.64-0.036 \cdot(\ln (T))^{2}-2.91 \cdot T^{0.5}\right) & \mathrm{T}<1.4 \mathrm{GeV} \\ 685 \times \exp \left(3.22-2.78 \cdot \ln (T)-1.5 \cdot T^{-1}\right) & \mathrm{T} \geq 1.4 \mathrm{GeV} .\end{cases}
$$

\subsubsection{LIS by Ghelfi et al. (2016): G16}

We used LIS by Ghelfi et al. $(2016,2017)$ (corrected in an erratum at https://arxiv.org/abs/1511.08650 (V3)) for the energy range below $800 \mathrm{GeV} /$ nucleon, using the best fit including Voyager data.

$$
\log _{10}\left(J_{\mathrm{LIS}_{\mathrm{G} 16}}(T)\right)=\sum_{j=0}^{14} c_{i} \times\left(\frac{\log _{10}(T)}{\log _{10}(800 \mathrm{GeV} / \text { nucleon })}\right)^{j}
$$

Coefficients $c_{i}$ are given in Table 3 of Ghelfi et al. (2016) (corrected in an erratum at https://arxiv.org/abs/ $1511.08650(\mathrm{~V} 3))$.

\subsubsection{LIS by Boschini et al. (2017): B17}

We used LIS by Boschini et al. (2017) given in Table 6 therein.

2.2.8. LIS by Garcia-Munoz et al. (1975): GM75

This LIS has been historically widely used in studies related to cosmogenic isotopes, even until recently (e.g., Steinhilber et al., 2012). Thus, we also consider it here.

$$
J_{\mathrm{LIS}_{\mathrm{GM} 75}}(T)=1.11 \cdot 10^{4}(T+0.78 \cdot \exp (0.25 \cdot T))^{-2.65} .
$$

2.2.9. LIS by Burger et al. (2000): B00

The LIS by Burger et al. (2000) is shown as parameterized by Usoskin et al. (2005):

$$
J_{\mathrm{LIS}_{\mathrm{U} 05}}(T)=\frac{1.9 \cdot 10^{4} \cdot P(T)^{-2.78}}{1+0.4866 \cdot P(T)^{-2.51}},
$$


where $P(T)=\sqrt{T\left(T+2 T_{\mathrm{r}}\right)}$ is the proton's rigidity. This LIS was used in many applications related to both cosmogenic isotopes and neutron monitor studies (e.g., Usoskin et al., 2011, 2016).

A comparison of the local interstellar spectra is shown in Figure 1, as normalized to the B00 one. They all, except for GM75, agree in the high-energy part but diverge by a factor of up to 2 in the energy range below several GeV. Accordingly, this uncertainty may influence the determination of the modulation potential.

It is important to consider not only protons but also $\alpha$ particles and heavier species of CR. While they are not dominant in GCR in the number of particles, the nucleonic number is essential and, also because of their less effective modulation in the heliosphere and magnetosphere, their contribution to the ground-based detectors is significant, being 30-50\% (Usoskin et al., 2011; Webber \& Higbie, 2003). Here we consider $\alpha$ particles (effectively including also heavier species) in LIS as 0.3 nucleonic ratio to protons with the given energy per nucleon. Although some models (BP16, G16, and B17) provide LIS for $\alpha$ in an explicit way, we used, for consistency, only proton spectrum scaled for heavier species, also in these cases. Since the single-parameter way of describing the GCR spectrum is the main advantage of the force-field parametrization, we keep it as simple as possible, viz., assuming heavier species to have the same (scaled) LIS as protons. Although this may be not exactly correct, we do it for consistency, keeping in mind that the values of $\phi$ have no physical meaning.

\section{Cosmic Ray Measurements by Energy-Integrating Methods}

Because of the thick Earth's atmosphere where an atmospheric nucleonic-muon-electromagnetic cascade is developed, ground-based detectors register secondaries of the cascade and cannot directly resolve the energy of the primary CR particles.

\subsection{Neutron Monitor}

The most useful ground-based detector to study heliospheric modulation of cosmic rays is a neutron monitor (NM). It was invented by John Simpson (Simpson, 1958; Simpson et al., 1953) for the International Geophysical Year (IGY), and several IGY-NMs were installed at different locations around the world in the 1950s. The design of NM was greatly improved by the International Quiet Sun Year in 1964, with the development of the NM64-type NM (Hatton \& Carimichael, 1964), which is presently a standard ground-based cosmic ray detector. A global network of standard NM instruments consists of several tens (up to 70) station distributed around the globe (see, e.g., the WDC-C, http://cidas.isee.nagoya-u.ac.jp/WDCCR/ or NMDB http://nmdb. eu databases) since the 1950s, and the data from the network are used to assess GCR variability in the energy range of 1-100 GeV (Belov, 2000; Dorman, 2004; Shea \& Smart, 2000).

A neutron monitor records count rate caused by the local flux of secondary particles that is an integral response to the entire $C R$ energy spectrum, weighted with the specific yield function of the detector:

$$
N(t)=\sum_{i=1}^{m} \int_{0}^{\infty} Y_{\mathrm{eff}_{\mathrm{NM}}, i}(T) \cdot J_{i}(T, t) \mathrm{d} T,
$$

where

$$
Y_{\mathrm{eff}_{\mathrm{NM}}, i}=Y_{i}(T) \cdot \kappa_{i}(T, M, \lambda)
$$

$m$ is the number of species of the primary CR (protons, $\alpha$ particles and heavier nuclei), $J(T, t)$ is the energy spectrum of CR outside the Earth's magnetosphere, and $Y(T)$ is the yield function of a NM, which depends of kinetic energy, $T$, of the primary CR particles. The parameter $\kappa_{i}$ is related to the geomagnetic cutoff, for a particle of type $i$, for the given geomagnetic latitude $\lambda$ and dipole moment $M$ :

$$
\kappa_{i}(T, M, \lambda)=\left\{\begin{array}{l}
0, T<T_{c, i} \\
1, T \geq T_{c, i}
\end{array}\right.
$$

where $T_{c, i}$ is the CR particle's kinetic energy corresponding to the geomagnetic cutoff rigidity in the NM location $P_{c}$ as

$$
T_{c, i}=\sqrt{\left(\frac{Z_{i}}{A_{i}}\right)^{2} P_{c}^{2}+T_{r}^{2}}-T_{r},
$$




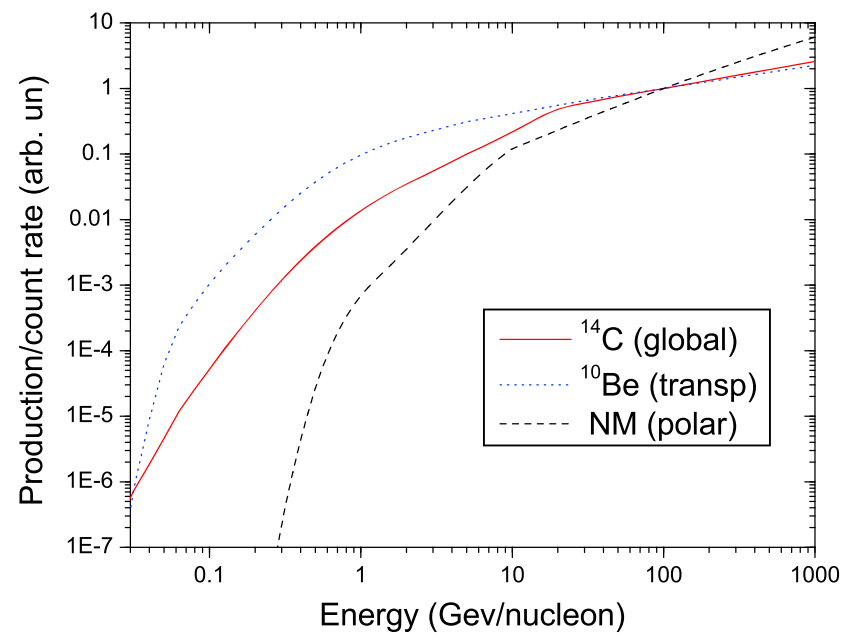

Figure 2. Yield functions of a standard sea level polar neutron monitor (black dashed curve) (Mishev et al., 2013), transport/deposition (Heikkilä et al., 2009) of ${ }^{10} \mathrm{Be}$ (blue dotted curve) and global production of ${ }^{14} \mathrm{C}$, as functions of the primary proton energy, for the geomagnetic dipole moment $M=8 \cdot 10^{22} \mathrm{~A} \mathrm{~m}^{2}$. Production yields of both ${ }^{10} \mathrm{Be}$ and ${ }^{14} \mathrm{C}$ were taken from Poluianov et al. (2016). All functions are shown for protons and normalized to unity at energy of $100 \mathrm{GeV}$. Tabulated yield functions for ${ }^{10} \mathrm{Be}$ and ${ }^{14} \mathrm{C}$ are given, for both protons and $\alpha$ particles and for different values of $M$, in the supporting information. where $P_{\mathrm{c}}$ (in gigavolt) is defined by the Stormer's formula (Elsasser et al., 1956) for a given $\lambda$ and $M$ (in units of $10^{22} \mathrm{~A} \mathrm{~m}^{2}$ ) as

$$
P_{\mathrm{c}} \approx 1.9 \cdot M \cdot \cos ^{4} \lambda \text {. }
$$

More details on the formalism can be found, for example, in Usoskin et al. (2005).

The yield function for a NM64 was calculated elsewhere (e.g., Clem and Dorman, 2000; Mangeard, Ruffolo, Sáiz, Nuntiyakul, et al., 2016; Mishev et al., 2013). Here we use the sea level NM yield function by Mishev et al. (2013) (including the data in the erratum) which account for the effective lateral spread of the atmospheric nucleonic cascade and the dead time of the detector. This yield function provides a good agreement with the data of NM latitudinal surveys (Gil et al., 2015). We note that most of the earlier NM yield functions (e.g., Clem \& Dorman, 2000) cannot reproduce the observed data on NM latitudinal survey (Caballero-Lopez \& Moraal, 2012; Gil et al., 2015) and are not considered here. Another recent yield function (Mangeard, Ruffolo, Sáiz, Madlee, et al., 2016; Mangeard, Ruffolo, Sáiz, Nuntiyakul, et al., 2016) agrees with the latitudinal survey and lies very close to that of Mishev et al. (2013) (including the data in the erratum) and would not alter the results presented here. The yield function for protons is shown in Figure 2 . One can see that protons with energy below $\approx 500 \mathrm{MeV}$ do not contribute to the NM count rate. This is called the "atmospheric cutoff."

\subsection{Globally Mixed ${ }^{14} \mathrm{C}$}

Radiocarbon ${ }^{14} \mathrm{C}$ is produced in the atmosphere via capture ( $n p$ reaction) of thermal neutrons by nitrogen, forming the main sink for neutrons in the atmosphere. These secondary neutrons are produced as a result of the nucleonic cascade initiated by energetic cosmic rays in the atmosphere. After production, radiocarbon gets oxidized to carbon dioxide and takes part, as gas, in the global carbon cycle (e.g., Damon et al., 1978). Because of that, it is globally mixed in the atmosphere so that wherever the tree to measure ${ }^{14} \mathrm{C}$ in was growing, the amount of the isotope should be roughly the same. One can obtain, from the measured ${ }^{14} \mathrm{C}$ abundance, using the carbon cycle model, the radiocarbon production rate $Q(t)$ in the atmosphere (e.g., Roth \& Joos, 2013).

The production rate (per unit area) of the globally mixed ${ }^{14} \mathrm{C}$ can be described as

$$
Q(t)=\sum_{i=1}^{m} \int_{0}^{\pi / 2} \int_{T_{c_{i}(\lambda)}}^{\infty} Y_{\mathrm{c}, i}(T) \cdot J_{i}(T, t) \cdot \cos \lambda \cdot \mathrm{d} T d \lambda,
$$

where $Y_{c}$ is the columnar yield function, defined as the number of ${ }^{14} \mathrm{C}$ atoms produced in the entire atmospheric column in the polar region by primary cosmic ray particles with unit intensity and fixed energy $T$ :

$$
Y_{\mathrm{c}}(T)=\int_{0}^{\mathrm{h}_{0}} Y(T, h) \cdot \mathrm{d} h,
$$

where $h_{0}=1033 \mathrm{~g} / \mathrm{cm}^{2}$ is the atmospheric depth at the sea level and $Y(T, h)$ is the yield function at a given height $h$ (Poluianov et al., 2016).

Since the GCR spectrum $J_{i}$ does not depend on latitude beyond the geomagnetosphere, the variables can be divided to rewrite equation (14) in the following form:

$$
Q(t)=\sum_{i=1}^{m} \int_{0}^{\infty} Y_{\mathrm{eff}_{14 \mathrm{C}}, i}(T) \cdot J_{i}(T, t) \cdot \mathrm{d} T,
$$

where

$$
Y_{\mathrm{eff}_{14 \mathrm{C}}, i}(T)=\int_{0}^{\pi / 2} \int_{0}^{\mathrm{h}_{0}} \kappa_{i}(T, M, \lambda) \cdot Y_{i}(T, h) \cdot \cos \lambda \cdot \mathrm{d} h \mathrm{~d} \lambda
$$

is the effective globally averaged yield function of ${ }^{14} \mathrm{C}$ production, calculated as an average, over the globe, of the columnar yield function $Y_{c, i}$. Since the globe is symmetric in the sense of ${ }^{14} \mathrm{C}$ production, integration can be made over one hemisphere only. 
After integration and assuming the dipole geomagnetic field (equation (13), the effective global yield function can be written as (Kovaltsov et al., 2012) follows:

$$
Y_{\mathrm{eff}_{14 c}, i}(T)=Y_{\mathrm{c}, i} \cdot(1-f(T))
$$

where

$$
f(T)= \begin{cases}\sqrt{1-\sqrt{P(T) /(1.9 M)}}, & P \leq 1.9 M \\ 0, & P>1.9 M\end{cases}
$$

The effective yield function for ${ }^{14} \mathrm{C}$ global production, $Y_{\text {eff }},{ }_{14},{ }^{\prime}$ is presented in the supporting information for protons and $\alpha$ particles (effectively including also the heavier species) and for different values of the geomagnetic dipole moment $M$. An example of $Y_{\text {eff }_{14 C}}$ for protons and $M=8 \cdot 10^{22} \mathrm{~A} \mathrm{~m}^{2}$ (close to the modern value) is shown in Figure 2. One can see that radiocarbon is sensitive to less energetic cosmic rays than NM, responding significantly also to those with energy around $100 \mathrm{MeV}$. These less energetic particles still can produce sufficient amount of secondary neutrons in the stratosphere.

\subsection{Partly Mixed ${ }^{10} \mathrm{Be}$}

Cosmogenic isotope ${ }^{10} \mathrm{Be}$ is produced as a result of spallation of nitrogen and oxygen by products of the nucleonic cascade in the atmosphere. Upon production, beryllium atoms are believed to be attached to atmospheric aerosols and follow their fate. Since gravitational sedimentation and wet/dry deposition play an important role in beryllium transport, ${ }^{10} \mathrm{Be}$ is not globally mixed in the atmosphere but follows a complicated transport/deposition process which can be affected by regional climate, particularly in Greenland. Modern atmospheric dynamical models are able to reasonably reproduce the beryllium transport (e.g., Heikkilä et al., 2009; Sukhodolov et al., 2017; Usoskin et al., 2009). The realistic transport/deposition process of beryllium isotopes was successfully parameterized by Heikkilä et al. (2009) via transport coefficients of seven large atmospheric "boxes" (stratosphere and six tropospheric boxes). Here we use this parametrization.

The depositional rate of ${ }^{10} \mathrm{Be}$ in polar region (Greenland or Antarctica) can be described as follows:

$$
\begin{aligned}
D(t)= & \sum_{i=1}^{m} \int_{-\pi / 2}^{\pi / 2} \int_{T_{c_{i}}}^{\infty} J_{i}(T, t) \cdot\left(f_{\mathrm{s}} \cdot Y_{\mathrm{s}, i}(T, \lambda)+\right. \\
& \left.+f_{\mathrm{t}}(\lambda) \cdot Y_{\mathrm{t}, i}(T, \lambda)\right) \cdot \cos \lambda \cdot \mathrm{d} T d \lambda,
\end{aligned}
$$

where $Y_{\mathrm{s}}$ and $Y_{\mathrm{t}}$ are similar to the columnar yield functions but calculated for stratospheric and tropospheric parts of the atmospheric column, respectively. Quantities $f_{\mathrm{s}}$ and $f_{\mathrm{t}}(\lambda)$ denote the transport coefficients for stratospheric and tropospheric production of ${ }^{10} \mathrm{Be}$, respectively, and can be obtained from Heikkilä et al. (2009). It should be noted that the height of the tropopause (the boundary separating troposphere and stratosphere) depends on the geographical latitude and season.

Similarly to radiocarbon, one can introduce the effective yield function also for ${ }^{10} \mathrm{Be}$ :

$$
\begin{aligned}
Y_{\text {eff }_{10 \mathrm{~B}}, i}(T)= & \int_{-\pi / 2}^{\pi / 2} \kappa_{i}(T, M, \lambda)\left(f_{\mathrm{s}} \int_{0}^{\mathrm{h}_{\mathrm{t}}(\lambda)} Y_{i}(T, h) \mathrm{d} h+\right. \\
& \left.+f_{\mathrm{t}}(\lambda) \int_{\mathrm{h}_{\mathrm{t}}(\lambda)}^{\mathrm{h}_{0}} Y_{i}(T, h) \mathrm{d} h\right) \cos \lambda \cdot \mathrm{d} \lambda,
\end{aligned}
$$

where $h(\lambda)$ is the depth of the tropopause and $Y_{i}(T)$ taken from Poluianov et al. (2016). The value of $D$ can be calculated via $Y_{\text {eff }}{ }_{10 \mathrm{Be}}, i$ similar to NM and ${ }^{14} \mathrm{C}$. Since ${ }^{10} \mathrm{Be}$ is not symmetrically deposited in Northern and Southern Hemispheres, integration is taken over the entire globe.

The effective yield function for ${ }^{10} \mathrm{Be}$ production is presented in the supporting information for protons and $\alpha$ particles (effectively including also the heavier species), separately for north and south polar regions and for different values of the geomagnetic dipole moment $M$. An example of the effective yield function for protons and $M=8 \cdot 10^{22} \mathrm{~A} \mathrm{~m}^{2}$ is shown in Figure 2. Beryllium is even more sensitive to low-energy cosmic rays than radiocarbon. This is caused by the fact that while production of ${ }^{14} \mathrm{C}$ requires development of the nucleonic cascade, ${ }^{10} \mathrm{Be}$ can be produced by spallation (threshold of the order of $10 \mathrm{MeV}$ ) of atmospheric $\mathrm{N}$ and $\mathrm{O}$ directly by primary cosmic ray particles. 


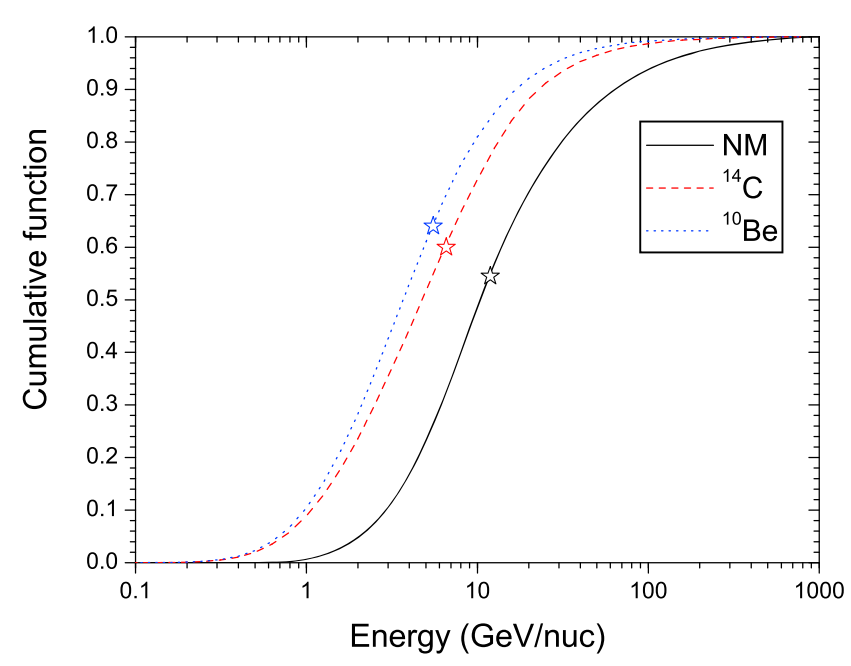

Figure 3. Cumulative function of the standard polar sea level NM count rate (black curve) and atmospheric production of ${ }^{14} \mathrm{C}$ (dashed red curve, global production) and polar deposition of ${ }^{10} \mathrm{Be}$ (dotted blue curve, global parameterized) for VP15 LIS, $M=8 \cdot 10^{22} \mathrm{~A} \mathrm{~m}^{2}$ and $\phi=500 \mathrm{MV}$. Colored stars denote the corresponding effective energy as calculated here.

\subsection{Cumulative Response Function}

Converting the effective yield function with the GCR spectrum, one can obtain the cumulative response function of a detector (cf. equation (9):

$$
\mathrm{CF}(\phi, T)=\frac{1}{N(\phi)} \sum_{i=1}^{m} \int_{0}^{T} Y_{\mathrm{eff}, i}\left(T^{\prime}\right) \cdot J_{i}\left(T^{\prime}, \phi\right) \cdot \mathrm{d} T^{\prime} .
$$

Examples of the cumulative response functions are shown in Figure 3 for the polar NM, global ${ }^{14} \mathrm{C}$, and polar deposited ${ }^{10} \mathrm{Be}$ for a specific condition as denoted in the figure caption. One can see that the NM is most sensitive to GCR with energy range 3-100 GeV/nucleon, while this range is smaller (1-30 GeV/nucleon) for cosmogenic isotopes. The exact curves slightly depend on the modulation level and the geomagnetic field.

\section{Effective Energy}

Since different detectors have different yield (response) functions to primary cosmic rays (Figure 2), it is practical to use the concept of effective energy as, for example, NMs have lower effective energy than muon detectors. Sometimes fixed energy (10 GV for a NM) (see Belov, 2000), median energy (Ahluwalia \& Fikani, 2007; Jämsén et al., 2007), maximum of the differential response function (McCracken et al., 2004), or the integral effective energy (Alanko et al., 2003) are used to characterize the detector's response for a NM. However, the concept of effective energy is not clear, as, for example, the median energy, which halves the cumulative response function (Figure 3), may vary within the solar cycle. Here we define the effective energy as the energy at which the variability of the GCR flux is directly proportional to that of the detector's count rate, so that the percentage variability of the detector's count rate is equal to that of the GCR flux at this energy.

In order to illustrate this, we show in Figure 4 variability of the count rates of Oulu NM for the period 1993-2016, along with the variability (over the same period) of the GCR proton flux at fixed energies of 6, 11.9 , and $24 \mathrm{GeV}$. The latter was calculated using the force-field approximation with VP15 LIS and the values of $\phi$ obtained from Usoskin et al. (2017). The higher-energy proton flux ( $24 \mathrm{GeV})$ varies too weak $( \pm 4 \%)$ and the lower energy proton flux - too strong $( \pm 15 \%)$ versus variability of the observed Oulu NM count rate $( \pm 10 \%)$. On the other hand, protons with $11.9 \mathrm{GeV}$ energy almost exactly correspond to the Oulu NM data variability.

In order to quantify this we define, as the effective energy $T_{\text {eff, }}$ energy which keeps the ratio $R(T, \phi)=$ $N(\phi) / J(T, \phi)$ constant within the range of $\phi=200-1,500 \mathrm{MV}$, which covers the range of the modulation potential from deep minima to periods of very high activity. This is illustrated in Figure 5 , which shows the ratio (for the standard polar NM) for different energies. For $T_{\text {eff }}=11.7 \mathrm{GeV} /$ nucleon, the ratio is constant within about $\pm 1 \%$, while too high or too low energy leads to an essential trend in the relation. This value is considered as the effective energy for a polar NM.

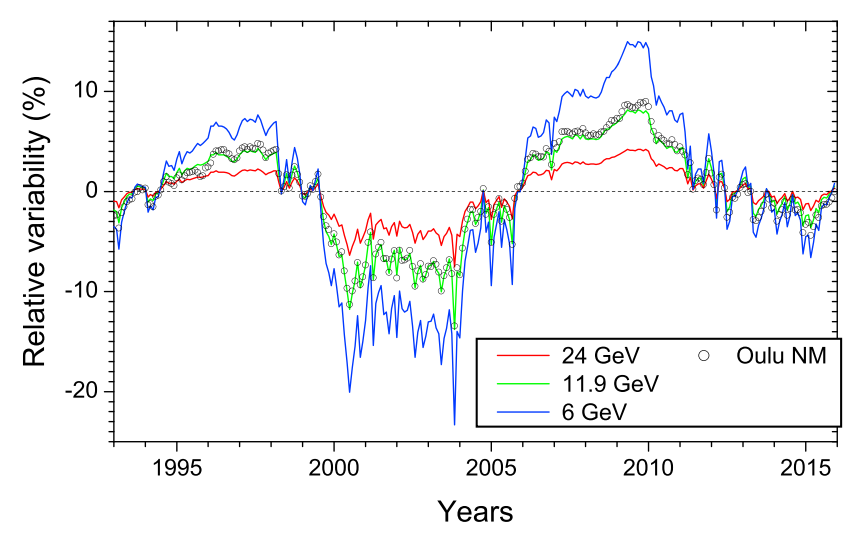

Figure 4. Relative variability (in percent to the mean level) for the period 1993-2016 of the Oulu NM (dots) and GCR at fixed energy (as denoted in the legend).
Correspondingly, the values of $E_{\text {eff }}$ can be calculated for sea level NMs with different geomagnetic rigidity cutoffs, as shown in Figure 6. Although the exact value of the effective energy slightly depends on the LIS model, this dependence is weak and accounts only for several percent.

The effective energy can be defined in a similar way also for cosmogenic isotopes. $E_{\text {eff }}$ for the global ${ }^{14} \mathrm{C}$ was found to be $6-7 \mathrm{GeV}$ (see Figure 3) depending on the exact geomagnetic dipole moment $M$ (in the range $6-1210^{22} \mathrm{~A} \mathrm{~m}^{2}$ ) and LIS models. The effective energy for ${ }^{14} \mathrm{C}$ is nearly half of that for the NM implying that radiocarbon is sensitive to less energetic cosmic rays than even a polar NM. The effective energy for the realistic production, transport, and deposition of ${ }^{10} \mathrm{Be}$ is $5.3-6.2 \mathrm{GeV}$ depending on the exact $M$ values and LIS models, which is slightly lower than that for ${ }^{14} \mathrm{C}$. The values of the effective energy for the modern conditions $\left(M=8 \cdot 10^{22} \mathrm{~A} \mathrm{~m}^{2}\right)$ are shown in Figure 3. One can see that they are somewhat higher than the median energy; thus, the median energy tends to underestimate the effective energy of a detector. 


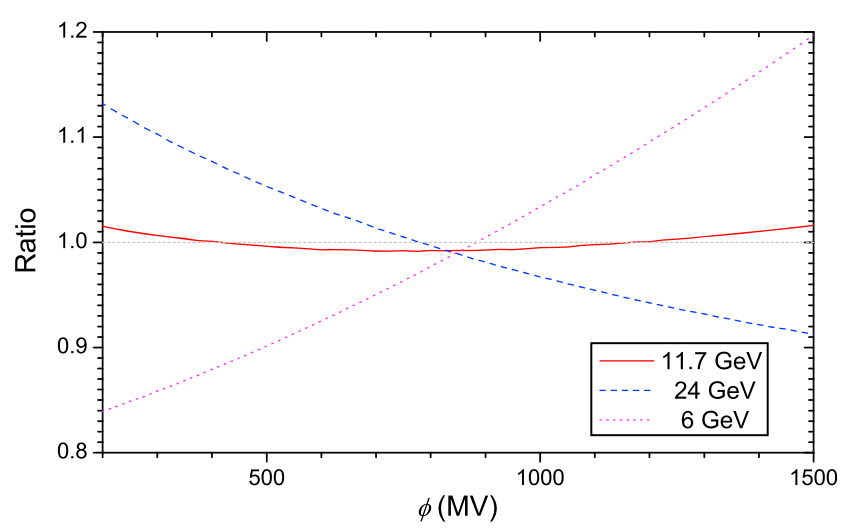

Figure 5. Ratio of the polar NM count rate to the flux of GCR protons with given energy (as denoted in the legend) as function of the modulation potential $\phi$, for the VP15 model of LIS. All curves are normalized to the unity in the range of $200-1,500 \mathrm{MV}$, as indicated by the horizontal dashed line.
We emphasize that this effective energy applies only to GCR variability. The effective energy for SEPs is significantly lower, of the order (Integral effective energy was used in these works.) of $200 \mathrm{MeV}$ for ${ }^{14} \mathrm{C}$ and ${ }^{10} \mathrm{Be}$ (Kovaltsov et al., 2014) and $800 \mathrm{MeV}$ for NM (Asvestari et al., 2017), because of much softer energy spectrum of SEP.

\section{Modulation Potential for Different Local Interstellar Spectra}

Since the LIS shape is uncertain (see section 2.2), the exact value of the modulation potential depends on the selected LIS. This means that the same modulated spectrum of GCR in the vicinity of Earth can be parameterized by the force-field approximation using different values of $\phi$ when based on different LIS models. This leads to an uncertainty in the absolute value of $\phi$, which makes little sense without specifying the LIS model employed. For example, the values of $\phi$ reconstructed by Steinhilber et al. (2012) from cosmogenic isotope data cannot be directly compared with the modulation potential calculated from the world NM network data by Usoskin et al. (2011, 2017) for the last decades, because the former are based in GM75 LIS, while the latter on B00 and VP15 LIS. Moreover, the $\phi$ series need a recalibration, if a newer LIS model is used.

A good news is that the modulation potential can be converted from one LIS to the other using an ad hoc relation. This is typically done using a linear relation (Herbst et al., 2010, 2017; Usoskin et al., 2005), so that $\phi$ computed for one LIS (e.g., B00) can be with high accuracy recalculated to another LIS linearly as

$$
\phi_{\mathrm{LIS}}=A \cdot \phi_{\mathrm{B} 00}+B
$$

There is no a priori reason to expect the relation to be linear, but it just appears so making the linear form sufficient to parameterize the relation. Usually, such conversion is done by refitting the modulated GCR proton spectra in a prescribed energy range using the force-field approximation with another LIS (Herbst et al., 2017; Usoskin et al., 2005). However, this method has some disadvantages. Refitting of the spectra is made in a given energy range without considering the exact way the modulation potential was computed. For example, such fit is greatly affected by the low-energy range of the spectrum, below a few GeV/nucleon, where the effect of modulation is stronger, but this range is below the effective energy of a detector (see section 4) and gives relatively low contribution to the detector's yield. Values of $\phi$ are typically calculated from the NM network data (e.g., Usoskin et al., 2005), which is responsive to higher-energy range ( $E_{\text {eff }} \approx 12 \mathrm{GeV} /$ nucleon for a polar $\mathrm{NM}$ ) and is almost insensitive to the energy range below $1 \mathrm{GeV} /$ nucleon. However, the modulation potential for farther past can only be reconstructed using the data of cosmogenic isotopes (Usoskin, 2017) whose production is sensitive to lower energy range than NM ( $E_{\text {eff }} \approx 6 \mathrm{GeV} /$ nucleon). Accordingly, the conversion should account for the exact method of $\phi$ computation and can appear different for different methods. Here we propose a new way to convert $\phi$ values, which is free of both shortcomings discussed above.

Figure 7 shows the relation between the count rate of a standard polar NM, calculated using equation (9), and the modulation potential for different LIS models. All curves differ from each other, particularly so for the BP16 LIS. This implies that the same count rate of a NM corresponds to different values of $\phi$ when different LIS models are used. This is illustrated in Figure 8 , where one can see that the same count rate $(15 \mathrm{c} / \mathrm{s})$ of the standard NM corresponds to different values of $\phi$ when different LIS models are used. In the shown case, the difference in the values of the modulation potential defined for $\mathrm{B} 00$ and BP16 LIS models is as large as $265 \mathrm{MV}$. We scanned it over the range of the NM count rates from 9 to $16 \mathrm{c} / \mathrm{s}$, which corresponds to the range between the maximum of the highest solar cycles and the Maunder minimum (Usoskin et al., 2002). The plot of the corresponding $\phi$ values obtained (as shown in
Figure 6. Dependence of the effective energy $E_{\text {eff }}$ of a sea level NM64 on the geomagnetic rigidity cutoff for different LIS models as denoted in the legend. 


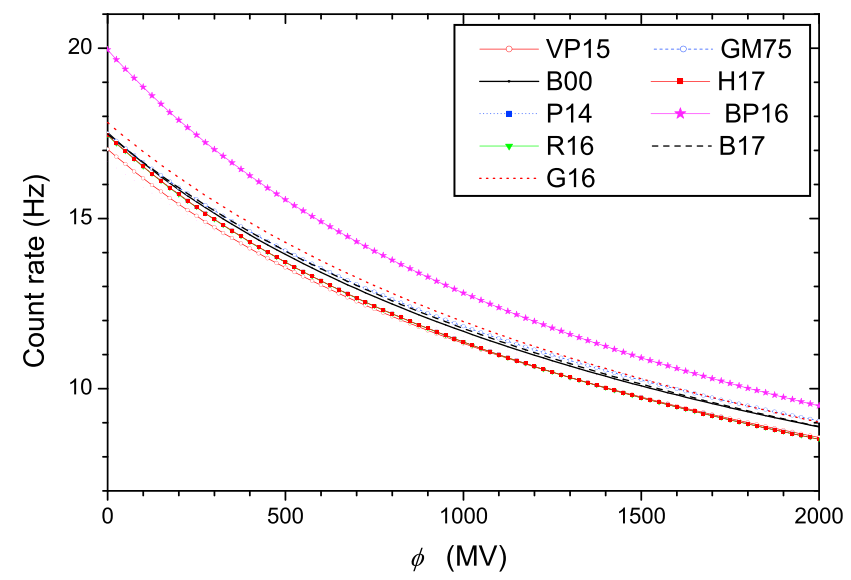

Figure 7. The count rate of the standard NM (1-NM64 at the sea level in the polar region) as function of the modulation potential $\phi$ for different LIS (denoted in the legend) analyzed here.

Figure 8) using B00 and BP16 LIS models is shown in Figure 9 as red dots. The relation is almost perfectly linear and can be approximated by formula (25) with coefficients $A=1.014$ and $B=261 \mathrm{MV}$. This comparison was done for other LIS models as well, and the results are collected in the first block of Table 1.

A similar comparison was made using global production for ${ }^{14} \mathrm{C}$ and polar deposition for ${ }^{10} \mathrm{Be}$. The results are also summarized in Table 1. The value of the linear relation coefficients is shown for the geomagnetic dipole moment $M=8 \cdot 10^{22} \mathrm{~A} \mathrm{~m}^{2}$, but they are almost independent on the exact value of $M$. The values of $A$ and $B$ remain within $\pm 4 \cdot 10^{-3}$ and $\pm 1 \mathrm{MV}$, respectively, around the values shown in Table 1 , for the range of $M(6-12) \cdot 10^{22} \mathrm{~A} \mathrm{~m}^{2}$, which covers the range of the geomagnetic field variability during the last 10 millennia (Usoskin et al., 2016).

One can see from Table 1 that coefficients $A$ and $B$ are different for NM and cosmogenic isotopes, as caused by essential difference in the effective energy for these energy-integration detectors. We note that the results are quite close to each other for P14, R16, and H17, since these LISs differ from each other only in the lower energy range $<1 \mathrm{GeV}$, which is not dominant for the detectors.

The result of this method to reduction of $\phi$ values between various LISs differs from earlier methods based on refitting the spectra (Herbst et al., 2017; Usoskin et al., 2005). We note that with our method the values of the modulation potential are converted "absolutely," viz., the production/count/deposition rate is equalized

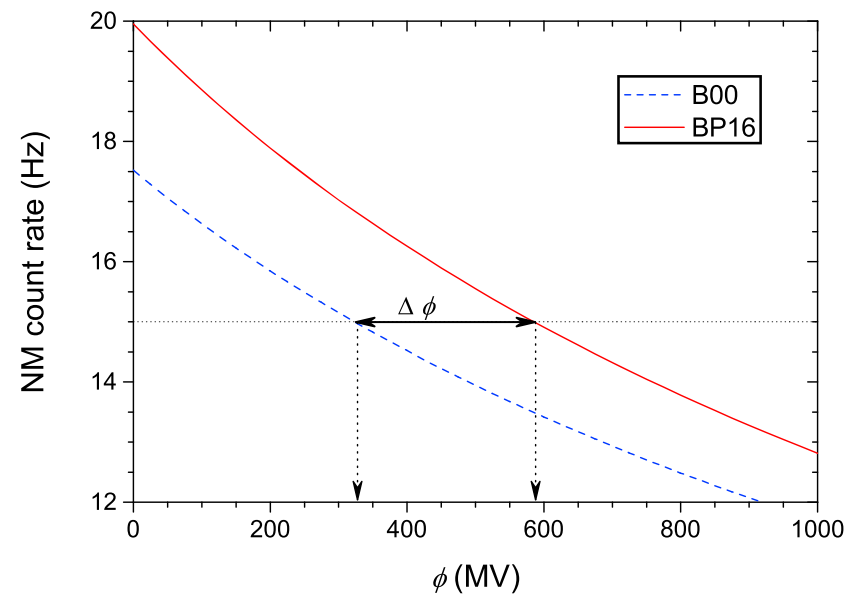

Figure 8. Illustration of the definition of the modulation potential difference $\Delta \phi$. For a given polar standard NM count rate (15 Hz here, horizontal line), the corresponding value of $\phi$ is calculated (vertical lines), and the difference between them is defined as $\Delta \phi$. This example is based on B00 and BP16 spectra and the polar NM. $\phi_{\mathrm{B} 00}=322 \mathrm{MV}$, $\phi_{\mathrm{BP} 16}=587 \mathrm{MV}$, and the corresponding $\Delta \phi=265 \mathrm{MV}$. 


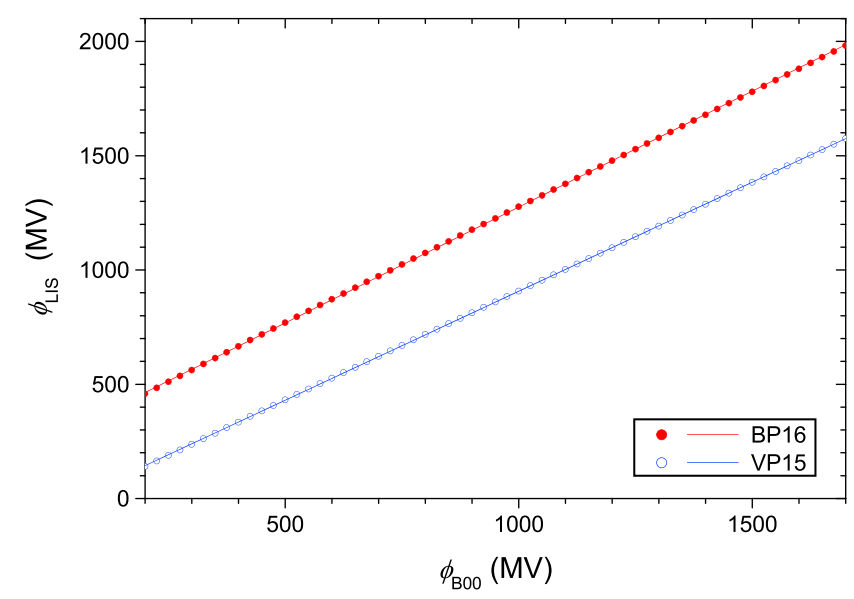

Figure 9. Relation between the $\phi_{\mathrm{B} 00}\left(X\right.$ axis) and $\phi_{\mathrm{LIS}}(Y$ axis), defined for a polar NM, for VP15 and BP16 spectra. The best fit linear relations (Table 1 ) are shown as lines.

between different LIS models. We have checked that the use of Table 1 for reduction of $\phi$ between different LIS models gives the accuracy (in terms of NM count rates or production/deposition rates of cosmogenic isotopes) within $0.5 \%$ which is much better than the accuracy of the spectral refitting method, which may reach $5-10 \%$ (Figure 5 in Herbst et al., 2017).

\section{Conclusions}

We have refined the method of assessment of the heliospheric modulation potential $\phi$ from measurements of ground-based neutron monitors for the recent decades and cosmogenic isotopes on longer timescales. While the modulation potential does not offer a physical meaning, it provides a handy and easy-to-use single-parameter parametrization of the spectra of GCR at the Earth's orbit. Three methods to assess the modulation potential are discussed here:

1. Ground-based polar neutron monitor is the main instrument to monitor GCR variability over more than 60 years.

2. Radiocarbon ${ }^{14} \mathrm{C}$ measured in tree rings is a globally averaged index of GCR flux on the timescales of up to 10 millennia.

3. Cosmogenic isotope ${ }^{10} \mathrm{Be}$ measured in polar ice cores is also an index of GCR flux on long-term scales.

Table 1

Coefficients $A$ and $B$ of Recalculation (Equation (25), Where $\phi$ Is Expressed in Megavolts) of the Modulation Potential $\phi$ From the B00 LIS to Other LISs Considered Here, for the Polar NM, Globally Mixed ${ }^{14} \mathrm{C}$ and ${ }^{10}$ Be Deposited in Southern Polar Region (as Described in the Text)

\begin{tabular}{|c|c|c|c|c|c|c|}
\hline \multirow[b]{2}{*}{ LIS } & \multicolumn{2}{|c|}{ Polar NM } & \multicolumn{2}{|c|}{ Global ${ }^{14} \mathrm{C}$} & \multicolumn{2}{|c|}{ Transport ${ }^{10} \mathrm{Be}$} \\
\hline & A & $B$ & A & $B$ & A & $B$ \\
\hline P14 & 0.921 & 0 & 0.968 & 39 & 0.981 & 43 \\
\hline VP15 & 0.954 & -47 & 0.984 & -29 & 0.991 & -27 \\
\hline BP16 & 1.014 & 261 & 1.081 & 201 & 1.081 & 196 \\
\hline R16 & 0.92 & -1 & 0.969 & 38 & 0.981 & 41 \\
\hline $\mathrm{H} 17$ & 0.921 & 0 & 0.968 & 40 & 0.981 & 44 \\
\hline G16 & 1.014 & 53 & 1.087 & -38 & 1.091 & -43 \\
\hline B17 & 1.007 & 14 & 1.071 & -58 & 1.072 & -63 \\
\hline GM75 & 1.041 & 1 & 1.03 & -48 & 1.027 & -48 \\
\hline
\end{tabular}

Note. All coefficients are given for the geomagnetic dipole moment $M=8$. $10^{22} \mathrm{~A} \mathrm{~m}^{2}$. Statistical uncertainties of the coefficients are better than 0.001 and $1 \mathrm{MV}$ for $A$ and $B$, respectively. 
Acknowledgments

The effective yield functions for cosmogenic ${ }^{14} \mathrm{C}$ (globally mixed in the atmosphere) and ${ }^{10} \mathrm{Be}$ (realistically deposited in the polar region) are provided, in a tabulated form, in the supporting information. This work was partially supported by the ReSoLVE Centre of Excellence (Academy of Finland, project 272157). A. G. acknowledges the Polish National Science Centre, decision DEC-2016/22/E/HS5/00406.
For the cosmogenic isotope method we provide, in the supporting information, effective yield functions, which makes it possible for a reader to directly calculate production/deposition rates using straightforward computations

We define the effective energy of GCR for each type of the measurement method. The effective energy appears to vary between 11 and $12 \mathrm{GeV} /$ nucleon for a polar NM and $33 \mathrm{GeV} /$ nucleon for an equatorial NM, implying that a NM is mostly responsive to the variability of middle-energy cosmic rays of several tens of GeV energy. Cosmogenic isotopes are sensitive to lower energy of GCR, the effective energy is found $5-6 \mathrm{GeV} /$ nucleon for

${ }^{10} \mathrm{Be}$ and 6-7 GeV/nucleon for ${ }^{14} \mathrm{C}$.

The exact value of $\phi$ depends on the selected model of LIS. Since there is a large uncertainty in the shape of LIS, especially in the lower energy range to which the detectors are mostly responsive, the values of the modulation potential need to be converted between different LIS models. A new approach of the modulation potential conversion is proposed, which considers the exact method of cosmic ray measurements. It is shown that the conversion relation is almost perfectly linear, and the coefficients for conversion are provided for different LIS models, methods, and the geomagnetic dipole moments.

This forms a basis for advanced and more precise studies of the cosmic ray and solar variability in the past using such methods as ground-based neutron monitors and cosmogenic isotopes and harmonization of different works related to variability of galactic cosmic ray flux in the vicinity of Earth, on long-term scale.

\section{References}

Abe, K., Fuke, H., Haino, S., Hams, T., Hasegawa, M., Horikoshi, A., ... Yoshimura, K. (2016). Measurements of cosmic-ray proton and helium spectra from the BESS-polar long-duration balloon flights over antarctica. The Astrophysical Journal, 822, 65. https://doi.org/10.3847/0004-637X/822/2/65

Adriani, O., Barbarino, G. C., Bazilevskaya, G. A., Bellotti, R., Boezio, M., Bogomolov, E. A., ... Vos, E. E. (2013). Time dependence of the proton flux measured by PAMELA during the 2006 July-2009 December solar minimum. The Astrophysical Journal, 765, 91. https://doi.org/10.1088/0004-637X/765/2/91

Aguilar, M., Aisa, D., Alpat, B., Alvino, A., Ambrosi, G., Andeen, K., ... Sun, W. H. (2015). Precision measurement of the proton flux in primary cosmic rays from rigidity $1 \mathrm{GV}$ to $1.8 \mathrm{TV}$ with the alpha magnetic spectrometer on the International Space Station. Physical Review Letters, 114(17), 171103. https://doi.org/10.1103/PhysRevLett.114.171103

Ahluwalia, H. S., \& Fikani, M. M. (2007). Cosmic ray detector response to transient solar modulation: Forbush decreases. Journal of Geophysical Research, 112, A08105. https://doi.org/10.1029/2006JA011958

Alanko, K., Usoskin, I. G., Mursula, K., \& Kovaltsov, G. A. (2003). Heliospheric modulation strength: Effective neutron monitor energy. Advances in Space Research, 32, 615-620. https://doi.org/10.1016/S0273-1177(03)00348-X

Alcaraz, J., Alpat, B., Ambrosi, G., Anderhub, H., Ao, L., Arefiev, A., ... Zimmermann, B. (2000). Cosmic protons. Physics Letters B, $490,27-35$. https://doi.org/10.1016/S0370-2693(00)00970-9

Asvestari, A., Willamo, T., Gil, A., Usoskin, I., Kovaltsov, G., Mikhailov, V., \& Mayorov, A. (2017). Analysis of ground level enhancements (GLE): Extreme solar energetic particle events have hard spectra. Advances in Space Research, 60, 781-787. https://doi.org/10.1016/j.asr.2016.08.043

Asvestari, E., Usoskin, I. G., Kovaltsov, G. A., Owens, M. J., Krivova, N. A., Rubinetti, S., \& Taricco, C. (2017). Assessment of different sunspot number series using the cosmogenic isotope ${ }^{44} \mathrm{Ti}$ in meteorites. Monthly Notices of the Royal Astronomical Society, 467, $1608-1613$. https://doi.org/10.1093/mnras/stx190

Beer, J., McCracken, K., \& von Steiger, R. (2012). Cosmogenic Radionuclides: Theory and Applications in the Terrestrial and Space Environments. Berlin: Springer.

Belov, A. (2000). Large scale modulation: View from the Earth. Space Science Reviews, 93, 79-105. https://doi.org/10.1023/A:1026584109817 Bisschoff, D., \& Potgieter, M. S. (2016). New local interstellar spectra for protons, helium and carbon derived from PAMELA and Voyager 1 observations. Astrophysics and Space Science, 361, 48. https: //doi.org/10.1007/s10509-015-2633-8

Boschini, M. J., Della Torre, S., Gervasi, M., Grandi, D., Jóhannesson, G., Kachelriess, M., .. Tacconi, M. (2017). Solution of heliospheric propagation: Unveiling the local interstellar spectra of cosmic-ray species. The Astrophysical Journal, 840, 115. https://doi.org/10.3847/1538-4357/aa6e4f

Burger, R., Potgieter, M., \& Heber, B. (2000). Rigidity dependence of cosmic ray proton latitudinal gradients measured by the Ulysses spacecraft: Implications for the diffusion tensor. Journal of Geophysical Research, 105, 27,447-27,456. https://doi.org/10.1029/2000JA000153

Caballero-Lopez, R., \& Moraal, H. (2004). Limitations of the force field equation to describe cosmic ray modulation. Journal of Geophysical Research, 109, A01101. https://doi.org/10.1029/2003JA010098

Caballero-Lopez, R., \& Moraal, H. (2012). Cosmic-ray yield and response functions in the atmosphere. Journal of Geophysical Research, 117, A12103. https://doi.org/10.1029/2012JA017794

Clem, J., \& Dorman, L. (2000). Neutron monitor response functions. Space Science Reviews, 93, 335-359. https://doi.org/10.1023/A:1026508915269

Damon, P., Lerman, J., \& Long, A. (1978). Temporal fluctuations of atmospheric ${ }^{14} \mathrm{C}$ : Causal factors and implications. Annual Review of Earth and Planetary Sciences, 6, 457-494. https://doi.org/10.1146/annurev.ea.06. 050178.002325

Dorman, L. (2004). Cosmic Rays in the Earth's Atmosphere and Underground. Dordrecht: Kluwer Academic Publishers.

Elsasser, W., Nay, E., \& Winkler, J. (1956). Cosmic-ray intensity and geomagnetism. Nature, 178, 1226-1227. https://doi.org/10.1038/1781226a0

Garcia-Munoz, M., Mason, G., \& Simpson, J. (1975). The anomalous ${ }^{4} \mathrm{He}$ component in the cosmic-ray spectrum at below approximately $50 \mathrm{MeV}$ per nucleon during 1972-1974. The Astrophysical Journal, 202, 265-275.

Ghelfi, A., Barao, F., Derome, L., \& Maurin, D. (2016). Non-parametric determination of $\mathrm{H}$ and He interstellar fluxes from cosmic-ray data. Astronomy and Astrophysics, 591, A94. https://doi.org/10.1051/0004-6361/201527852 
Ghelfi, A., Maurin, D., Cheminet, A., Derome, L., Hubert, G., \& Melot, F. (2017). Neutron monitors and muon detectors for solar modulation studies: 2. $\phi$ time series. Advances in Space Research, 60, 833-847. https://doi.org/10.1016/j.asr.2016.06.027

Gil, A., Usoskin, I. G., Kovaltsov, G. A., Mishev, A. L., Corti, C., \& Bindi, V. (2015). Can we properly model the neutron monitor count rate? Journal of Geophysical Research: Space Physics, 120, 7172-7178. https://doi.org/10.1002/2015JA021654

Gleeson, L., \& Axford, W. (1968). Solar modulation of galactic cosmic rays. The Astrophysical Journal, 154, $1011-1026$. https://doi.org/10.1086/149822

Hatton, C. J., \& Carimichael, H. (1964). Experimental investigation of the NM-64 neutron monitor. Canadian Journal of Physics, 42 , 2443-2472. https://doi.org/10.1139/p64-222

Heikkilä, U., Beer, J., \& Feichter, J. (2009). Meridional transport and deposition of atmospheric ${ }^{10}$ Be. Atmospheric Chemistry and Physics, 9 , 515-527. https://doi.org/10.5194/acp-9-515-2009

Herbst, K., Kopp, A., Heber, B., Steinhilber, F., Fichtner, H., Scherer, K., \& Matthiä, D. (2010). On the importance of the local interstellar spectrum for the solar modulation parameter. Journal of Geophysical Research, 115, D00I20. https://doi.org/10.1029/2009JD012557

Herbst, K., Muscheler, R., \& Heber, B. (2017). The new local interstellar spectra and their influence on the production rates of the cosmogenic radionuclides ${ }^{10} \mathrm{Be}$ and ${ }^{14} \mathrm{C}$. Journal of Geophysical Research: Space Physics, 122, 23-34. https://doi.org/10.1002/2016JA023207

Inceoglu, F., Simoniello, R., Knudsen, V. F., Karoff, C., Olsen, J., Turck-Chiéze, S., \& Jacobsen, B. H. (2015). Grand solar minima and maxima deduced from ${ }^{10} \mathrm{Be}$ and ${ }^{14} \mathrm{C}$ : Magnetic dynamo configuration and polarity reversal. Astronomy and Astrophysics, $577, \mathrm{~A} 20$. https://doi.org/10.1051/0004-6361/201424212

Jämsén, T., Usoskin, I. G., Räihä, T., Sarkamo, J., \& Kovaltsov, G. A. (2007). Case study of Forbush decreases: Energy dependence of the recovery. Advances in Space Research, 40, 342-347. https: //doi.org/10.1016/j.asr.2007.02.025

Jokipii, J. R., \& Kóta, J. (1995). Three-dimensional cosmic-ray simulations: Heliographic latitude and current-sheet tilt. Space Science Reviews, 72, 379-384. https://doi.org/10.1007/BF00768808

Jokipii, J. R., \& Levy, E. H. (1977). Effects of particle drifts on the solar modulation of galactic cosmic rays. The Astrophysical Journal Letters, 213, L85-L88. https://doi.org/10.1086/182415

Kovaltsov, G., Mishev, A., \& Usoskin, I. (2012). A new model of cosmogenic production of radiocarbon ${ }^{14} \mathrm{C}$ in the atmosphere. Earth and Planetary Science Letters, 337, 114-120. https://doi.org/10.1016/j.epsl.2012.05.036

Kovaltsov, G. A., Usoskin, I. G., Cliver, E. W., Dietrich, W. F., \& Tylka, A. J. (2014). Fluence ordering of solar energetic proton events using cosmogenic radionuclide data. Solar Physics, 289, 4691-4700. https://doi.org/10.1007/s11207-014-0606-7

Mangeard, P.-S., Ruffolo, D., Sáiz, A., Nuntiyakul, W., Bieber, J. W., Clem, J., ... Humble, J. E. (2016). Dependence of the neutron monitor count rate and time delay distribution on the rigidity spectrum of primary cosmic rays. Journal of Geophysical Research: Space Physics, 121, 11,620-11,636. https://doi.org/10.1002/2016JA023515

Mangeard, P.-S., Ruffolo, D., Sáiz, A., Madlee, S., \& Nutaro, T. (2016). Monte Carlo simulation of the neutron monitor yield function. Journal of Geophysical Research: Space Physics, 121, 7435-7448. https://doi.org/10.1002/2016JA022638

Maurin, D., Cheminet, A., Derome, L., Ghelfi, A., \& Hubert, G. (2015). Neutron monitors and muon detectors for solar modulation studies: Interstellar flux, yield function, and assessment of critical parameters in count rate calculations. Advances in Space Research, 55, 363-389. https://doi.org/10.1016/j.asr.2014.06.021

McCracken, K., \& Beer, J. (2007). Long-term changes in the cosmic ray intensity at Earth, 1428-2005. Journal of Geophysical Research, 112, A10101. https://doi.org/10.1029/2006JA012117

McCracken, K., McDonald, F., Beer, J., Raisbeck, G., \& Yiou, F. (2004). A phenomenological study of the long-term cosmic ray modulation, 850-1958 ad. Journal of Geophysical Research, 109, A12103. https: //doi.org/10.1029/2004JA010685

Michel, R., Leya, l., \& Borges, L. (1996). Production of cosmogenic nuclides in meteoroids: Accelerator experiments and model calculations to decipher the cosmic ray record in extraterrestrial matter. Nuclear Instruments and Methods in Physics Research Section B, 113, 434-444. https://doi.org/10.1016/0168-583X(95)01345-8

Mishev, A. L., Usoskin, I. G., \& Kovaltsov, G. A. (2013). Neutron monitor yield function: New improved computations. Journal of Geophysical Research: Space Physics, 118, 2783-2788. https://doi.org/10.1002/jgra.50325

Moskalenko, I. V., Strong, A. W., Ormes, J. F., \& Potgieter, M. S. (2002). Secondary antiprotons and propagation of cosmic rays in the galaxy and heliosphere. The Astrophysical Journal, 565, 280-296. https://doi.org/10.1086/324402

Pallé, E., Butler, C. J., \& O'Brien, K. (2004). The possible connection between ionization in the atmosphere by cosmic rays and low level clouds. Journal of Atmospheric and Solar-Terrestrial Physics, 66, 1779-1790. https://doi.org/10.1016/j.jastp.2004.07.041

Poluianov, S. V., Kovaltsov, G. A., Mishev, A. L., \& Usoskin, I. G. (2016). Production of cosmogenic isotopes ${ }^{7} \mathrm{Be},{ }^{10} \mathrm{Be},{ }^{14} \mathrm{C},{ }^{22} \mathrm{Na}$, and ${ }^{36} \mathrm{Cl}$ in the atmosphere: Altitudinal profiles of yield functions. Journal of Geophysical Research: Atmospheres, 121, 8125-8136. https://doi.org/10.1002/2016JD025034

Potgieter, M. (2013). Solar modulation of cosmic rays. Living Reviews in Solar Physics, 10, 3. https: //doi.org/10.12942/lrsp-2013-3

Potgieter, M. S., Vos, E. E., Boezio, M., De Simone, N., Di Felice, V., \& Formato, V. (2014). Modulation of galactic protons in the heliosphere during the unusual solar minimum of 2006 to 2009. Solar Physics, 289, 391-406. https://doi.org/10.1007/s11207-013-0324-6

Raath, J. L., Potgieter, M. S., Strauss, R. D., \& Kopp, A. (2016). The effects of magnetic field modifications on the solar modulation of cosmic rays with a SDE-based model. Advances in Space Research, 57, 1965-1977. https://doi.org/10.1016/j.asr.2016.01.017

Roth, R., \& Joos, F. (2013). A reconstruction of radiocarbon production and total solar irradiance from the Holocene ${ }^{14} \mathrm{C}_{\text {and }} \mathrm{CO}_{2}$ records: Implications of data and model uncertainties. Climate of the Past, 9, 1879-1909. https://doi.org/10.5194/cp-9-1879-2013

Shea, M. A., \& Smart, D. F. (2000). Fifty years of cosmic radiation data. Space Science Reviews, 93, 229-262. https://doi.org/10.1023/A:1026500713452

Simpson, J. A. (1958). Cosmic radiation neutron intensity monitor, Annals of the Int. Geophysical Year IV, Part VII (pp. 351). London: Pergamon Press.

Simpson, J. A., Fonger, W., \& Treiman, S. B. (1953). Cosmic radiation intensity-time variations and their origin. I. Neutron intensity variation method and meteorological factors. Physical Review, 90, 934-950. https://doi.org/10.1103/PhysRev.90.934

Steinhilber, F., Abreu, J., Beer, J., Brunner, I., Christl, M., Fischer, H., ... Wilhelms, F. (2012). 9,400 years of cosmic radiation and solar activity from ice cores and tree rings. Proceedings of the National Academy of Sciences of the United States, 109(16), 5967-5971. https://doi.org/10.1073/pnas.1118965109

Stone, E. C., Cummings, A. C., McDonald, F. B., Heikkila, B. C., Lal, N., \& Webber, W. R. (2013). Voyager 1 observes low-energy galactic cosmic rays in a region depleted of heliospheric ions. Science, 341, 150-153. https://doi.org/10.1126/science.1236408

Sukhodolov, T., Usoskin, I., Rozanov, E., Asvestari, E., Ball, W., Curran, M., ... Traversi, R. (2017). Atmospheric impacts of the strongest known solar particle storm of 775 AD. Scientific Reports, 7, 45,257. https://doi.org/10.1038/srep45257

Usoskin, I. G. (2017). A history of solar activity over millennia. Living Reviews in Solar Physics, 14, 3. https://doi.org/10.1007/s41116-017-0006-9 
Usoskin, I. G., Mursula, K., Solanki, S. K., Schüssler, M., \& Kovaltsov, G. A. (2002). A physical reconstruction of cosmic ray intensity since 1610. Journal of Geophysical Research, 107, 1374. https://doi.org/10.1029/2002JA009343

Usoskin, I. G., Alanko-Huotari, K., Kovaltsov, G. A., \& Mursula, K. (2005). Heliospheric modulation of cosmic rays: Monthly reconstruction for 1951-2004. Journal of Geophysical Research, 110, A12108. https: //doi.org/10.1029/2005JA011250

Usoskin, I. G., Field, C. V., Schmidt, G. A., Leppänen, A.-P., Aldahan, A., Kovaltsov, G. A., ... Ungar, R. K. (2009). Short-term production and synoptic influences on atmospheric ${ }^{7}$ Be concentrations. Journal of Geophysical Research, 114, D06108. https://doi.org/10.1029/2008JD011333

Usoskin, I. G., Bazilevskaya, G. A., \& Kovaltsov, G. A. (2011). Solar modulation parameter for cosmic rays since 1936 reconstructed from ground-based neutron monitors and ionization chambers. Journal of Geophysical Research, 116, A02104. https://doi.org/10.1029/2010JA016105

Usoskin, I. G., Gallet, Y., Lopes, F., Kovaltsov, G. A., \& Hulot, G. (2016). Solar activity during the Holocene: The Hallstatt cycle and its consequence for grand minima and maxim. Astronomy and Astrophysics, 587, A150. https://doi.org/10.1051/0004-6361/201527295

Usoskin, I. G., Gil, A., Kovaltsov, G. A., Mishev, A. L., \& Mikhailov, V. V. (2017). Heliospheric modulation of cosmic rays during the neutron monitor era: Calibration using PAMELA data for 2006-2010. Journal of Geophysical Research: Space Physics, 122, 3875-3887. https://doi.org/10.1002/2016JA023819

Vainio, R., Desorgher, L., Heynderickx, D., Storini, M., Flückiger, E., Horne, R. B., ... Usoskin, I. G. (2009). Dynamics of the Earth's particle radiation environment. Space Science Reviews, 147, 187-231. https://doi.org/10.1007/s11214-009-9496-7

Vos, E. E., \& Potgieter, M. S. (2015). New modeling of galactic proton modulation during the minimum of solar cycle 23/24. The Astrophysical Journal, 815, 119. https://doi.org/10.1088/0004-637X/815/2/119

Webber, W., \& Higbie, P. (2003). Production of cosmogenic be nuclei in the Earth's atmosphere by cosmic rays: Its dependence on solar modulation and the interstellar cosmic ray spectrum. Journal of Geophysical Research, 108, 1355. https://doi.org/10.1029/2003JA009863 